\title{
Velocity Driven N-order Stick Compensator and Slip Suppressor for Nonlinear Friction in an Oil-Seal-Mounted Geared Motor
}

\author{
Juan Padron $^{* a)} \quad$ Student Member, Kiyoshi Ohishi* Fellow \\ Yuki Yokokura* Member, Toshimasa Miyazaki* Member
}

(Manuscript received June 3, 2019)

\begin{abstract}
Geared motors require oil for lubrication; however, the oil may leak if no sealing is provided. One method for preventing leaking is to mount oil seals on the input and output sides of the gear. However, this increases the effects of nonlinear friction in the motor, causing stick-slip during velocity zero-crossings. This paper proposes a stick-slip suppression method based on a velocity-driven N-order stick compensator (VDNSC) and a slip suppressor. The proposed stick compensator is based on a motor-side normalization compensator (MNC) and works as an order-variable friction observer, while the proposed slip suppressor is based on velocity-switched high frequency damping. The validity of the proposed method is confirmed through experiments with low-velocity control.
\end{abstract}

Keywords: nonlinear friction, geared motor, disturbance observer, stick-slip, oil seal

\section{Introduction}

Geared electric motors combine the ease of control and reliability offered by electric motors with a gear system that provides a high output torque. However, gears require lubrication to prevent excessive wear owing to dry contact, and this oil may leak from the motor if no sealing is provided. Oil leaks may damage the motor and its surroundings, resulting in catastrophic consequences if they occur in contaminationsensitive environments, e.g., laboratories, food processing plants or hospitals. Mounting oil seals in a geared motor prevents leaking, but greatly increases the effects of nonlinear friction. This causes stick-slip during velocity zero-crossings and affects the motor performance.

Nonlinear friction compensation for electric motors has been widely studied for many decades, and its complicated nature has led to a great variety of proposals. Some of the many approaches attempted thus far are given as follows:

(1) Dither-based compensation: Dither signals combined with a disturbance observer to compensate for static and kinetic friction ${ }^{(1)}$.

(2) Model-based compensation: Model-based friction observers, e.g., ${ }^{(2)}$, where the LuGre model ${ }^{(3)}$ is used.

(3) Integral-action-based compensation: Variable gain integrators ${ }^{(4)}$ and adaptive friction compensators based on integrators ${ }^{(5)}$.

(4) Nonlinear-disturbance-observer-based compensation: e.g., adaptive disturbance observers ${ }^{(6)}$

Dither-based compensation introduces small vibrations into the motor which hinder precise motion. Model-based compensation tends to be complicated in implementation and poorly robust against parameter changes. Integral action may

a) Correspondence to: Juan Padron. E-mail: s175066@stn.naga okaut.ac.jp

* Nagaoka University of Technology

1603-1, Kamitomioka, Nagaoka, Niigata 940-2188, Japan accumulate error due to other disturbances in addition to friction and cause windup. Disturbance observers have the advantages of being robust and not requiring any friction model; however, they are mainly designed to work as a minor loop on the motor-side; in the case of geared motors, the torsiontorque term that is reflected by the load-side is detected as a disturbance and compensated, inducing resonance on the load.

Employing a torque sensor allows to separate load-side and motor-side disturbances. This fact was exploited in ${ }^{(7)}$, where a friction observer based on a joint-torque sensor is proposed. This approach is further refined in ${ }^{(8)}$, where the motor-side normalization compensator (MNC) is proposed as a friction observer that also compensates for motor-side parameter variation, making it a powerful tool that is applicable in many control schemes. However, the MNC is unsuitable for suppressing nonlinear friction, as its disturbance-rejection characteristic is not strong enough, producing stick-slip during velocity zero-crossings in oil-seal-mounted motors.

This paper proposes a stick-slip suppression method based on a velocity-driven $\mathrm{N}$-order stick compensator (VDNSC) and a slip suppressor. The VDNSC is based on an N-order MNC whose order is switched with the motor velocity, making it possible to only raise the disturbance rejection over low velocities, where the nonlinear friction is the largest. The slip suppressor is based on a velocity-triggered high-frequency damping that counteracts the temporary negative-damping effect given during the mixed-lubrication transitions of the motor (Stribeck effect), suppressing the slip phenomenon. This strategy does not require any friction model, accounts for parameter variation and is adaptable to many control schemes.

The validity of the proposed method was proved by lowvelocity control experiments. 


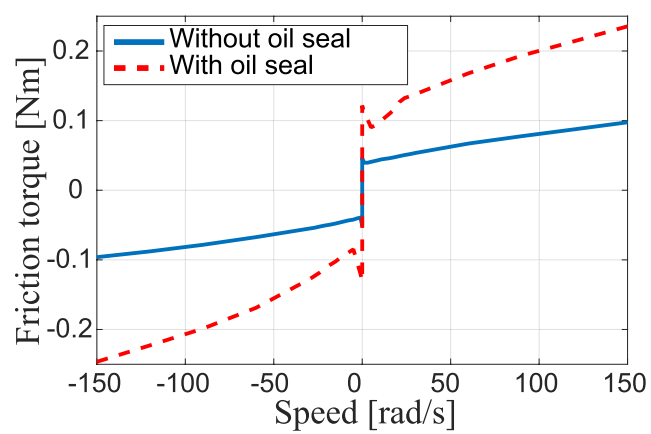

Fig. 1. Comparison of steady-state friction curve with and without oil seals in a geared motor

\section{Stick-Slip Phenomenon in Geared-Motor with Oil Seal}

The stick-slip phenomenon is characterized by a sudden brake followed by a sudden acceleration which is caused by strong nonlinear friction in the low-velocity region. The phenomenon is explained by its corresponding regions in the friction curve, as follows:

(1) Stick: The friction coefficient suddenly becomes very large, causing the motor to decelerate. This occurs in the pre-sliding (or boundary lubrication) zone, usually when the motor moves from a stop or switches direction slowly and the contact between the mechanical parts is still dominated by dry friction.

(2) Slip: The friction coefficient suddenly becomes negative, causing the motor to accelerate violently. This occurs in the slip (or mixed lubrication) zone, when the lubrication film begins to form and the friction abruptly decreases.

Figure 1 shows a comparison of the steady-state friction curves for two geared motors, one with oil seals mounted and one without sealing. The motor with oil seals has a much larger static friction and a larger, steeper gap between the static and kinetic friction zones. This causes stick-slip during velocity zero-crossings in the oil-seal-mounted motor, as seen in Fig. 2.

\section{Conventional Method for Stick-Slip Suppres- sion: The MNC}

3.1 Zero-order MNC The motor-side normalization compensator (MNC) is a friction observer based on the same design principle as the disturbance observer ${ }^{(9)}$ which employs information from the motor input current, velocity and torsion torque for estimation. Figure 3 shows a block diagram of the two-inertia system used to model the geared motor together with the conventional MNC and a P-velocity controller. $J_{m}, J_{l}, K_{t}, R_{g}$ and $K_{s}$ are the motor and load-side inertia, torque constant, gear ratio, and torsion stiffness, respectively, while $i_{c t r l}, \tau_{s}, \omega_{m}$ and $\tau_{d m}$ are the control input (controller reference), torsion torque, motor velocity, and nonlinear friction terms. The $n$ subscripts refer to nominal parameters.

The motor-side dynamics are given as

$$
J_{m} \dot{\omega}_{m}=K_{t} t_{q}^{r e f}-\frac{\tau_{s}}{R_{g}}-\tau_{d m}
$$

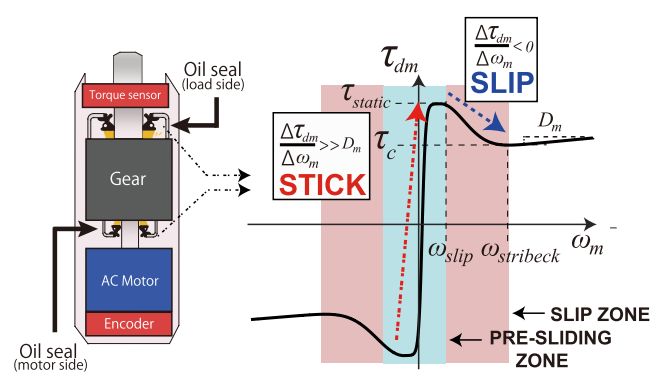

(a)

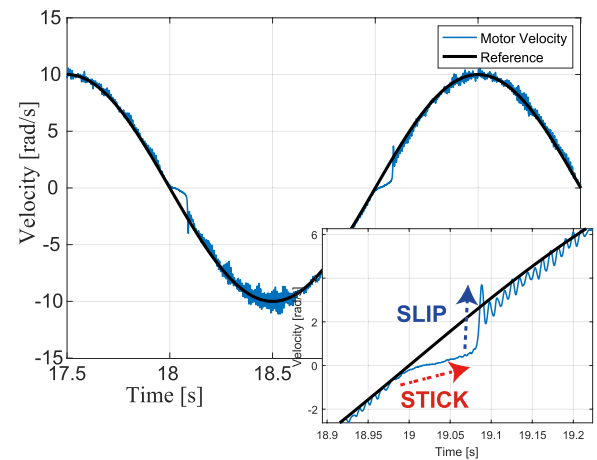

(b)

Fig. 2. Stick-slip in velocity response and its correspondence to the friction characteristics of the motor: (a) Friction curve; (b) Near zero velocity response

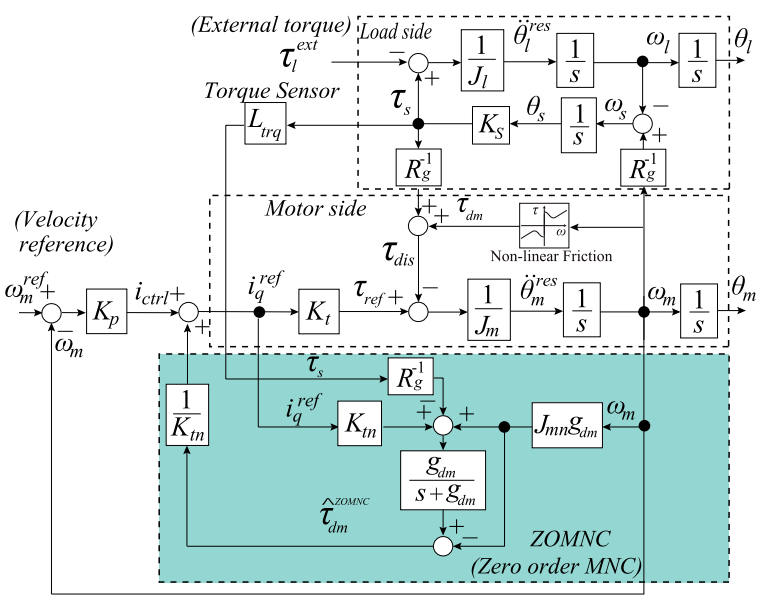

Fig. 3. Block diagram of conventional MNC (conventional method 1)

The conventional MNC is constructed by approximating the motor-side disturbance $\tau_{d m}$ (the disturbance term that excludes the torsion torque, in this case the motor friction) as a constant, as follows:

$$
\tau_{d m}^{Z O M N C}(t)=a_{0} . \quad \text { (Zero order approximation)... }
$$

This design, based on an approximation of the disturbance as a step function, is called a zero-order MNC (ZOMNC). For the ZOMNC, the disturbance value is estimated as a lowpass-filtered version of the original disturbance. It is given as

$$
\begin{aligned}
\hat{\tau}_{d m}^{Z O M N C} & =\frac{g_{d m}}{s+g_{d m}}\left(K_{t n} i_{q}^{r e f}-\frac{\tau_{s}}{R_{g}}-J_{m n} \omega_{m} s\right) \\
& =\frac{g_{d m}}{s+g_{d m}} \tau_{d m}, \ldots \ldots \ldots \ldots \ldots \ldots
\end{aligned}
$$

where $g_{d m}$ is the bandwidth of the low-pass-filter of the 


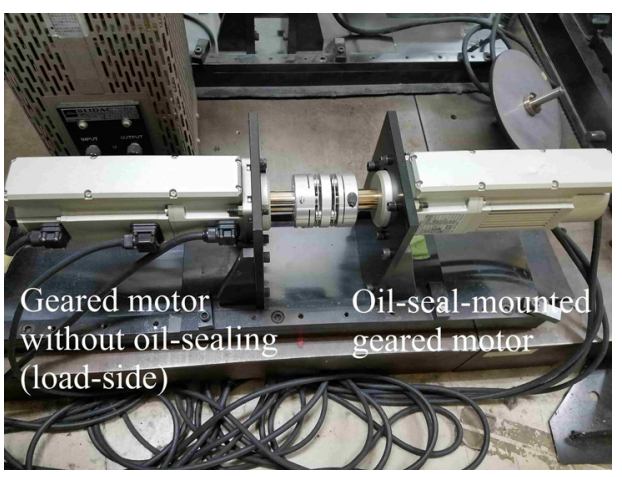

Fig. 4. Experimental equipment

Table 1. Experimental parameters

\begin{tabular}{l|c|l}
\hline Motor-side Inertia & $J_{m}$ & $2.86 \times 10^{-5} \mathrm{Kgm}^{2}$ \\
Load-side Inertia & $J_{l}$ & $0.147 \mathrm{Kgm}^{2}$ \\
Gear ratio & $R_{g}$ & 50 \\
Torque Constant & $K_{t}$ & $0.318 \mathrm{Nm} / \mathrm{A}$ \\
Torsion Spring Constant & $K_{s}$ & $3276.19 \mathrm{Nm} / \mathrm{rad}$ \\
Anti-resonance frequency & $\omega_{a}$ & $23.76[\mathrm{~Hz}]$ \\
Resonance frequency & $\omega_{r}$ & $41.58[\mathrm{~Hz}]$ \\
\hline P-velocity control bandwidth & $g_{p}$ & $120 \mathrm{rad} / \mathrm{s}$ \\
ZOMNC bandwidth & $g_{d m}$ & $400 \mathrm{rad} / \mathrm{s}$ \\
FOMNC poles & {$\left[g_{0}, g_{1}\right]$} & {$[-200,-200] \mathrm{rad} / \mathrm{s}$} \\
VDNSC poles & {$\left[g_{0}, g_{1}, g_{2}, g_{3}\right]$} & {$[-400,-200,-200,-200] \mathrm{rad} / \mathrm{s}$} \\
Slip suppressor damping gain & $K_{v}$ & 0.2 \\
Slip suppressor HPF cutoff frequency & $g_{d m p}$ & $2000 \mathrm{rad} / \mathrm{s}$ \\
Slip suppressor velocity threshold & $\omega_{t h l d}$ & $3 \mathrm{rad} / \mathrm{s}$ \\
\hline
\end{tabular}

\section{ZOMNC.}

3.2 Experimental Setting and Parameters Figure 4 shows the experimental setting used for this study, and is comprised of the oil-seal-mounted geared motor coupled with a geared motor without oil seal, the latter used as a load inertia. The measured plant and employed experimental parameters are given in Table 1.

A simple P-velocity control system is implemented to evaluate the performance of the different friction-compensation methods explained in this paper. A slow-changing sinusoidal velocity reference of $0.5 \mathrm{~Hz}$ is used, defined as follows:

$$
\omega_{m}^{r e f}=10 \sin (2 \pi \times 0.5 t) \quad \mathrm{rad} / \mathrm{s} .
$$

\subsection{Experimental Results: ZOMNC}

Figure 5 shows the experimental results for velocity control with the ZOMNC as a friction compensator.

It is clear that the ZOMNC effectively suppresses kinetic friction, as the velocity response follows the reference. However, it is not quick enough to overcome the nonlinear friction during zero-crossings, which results in the stick-slip phenomenon producing in the motor.

This result confirms that the conventional MNC works well for suppressing slow-changing, linear friction, but not for strong nonlinear friction. It has been demonstrated analytically that the ZOMNC disturbance-rejection characteristic is not suitable for this kind of friction ${ }^{(10)}$; thus, an alternative approach is required.

3.4 N-Order MNC Increasing the order of a disturbance observer enhances the disturbance rejection ${ }^{(11)}$. By applying the same methodology for the MNC design, friction is approximated as a $\mathrm{N}$-order polynomial as follows:

$$
\tau_{d m}^{N O M N C}(t)=a_{N} t^{N}+a_{N-1} t^{N-1}+\ldots+a_{0}
$$

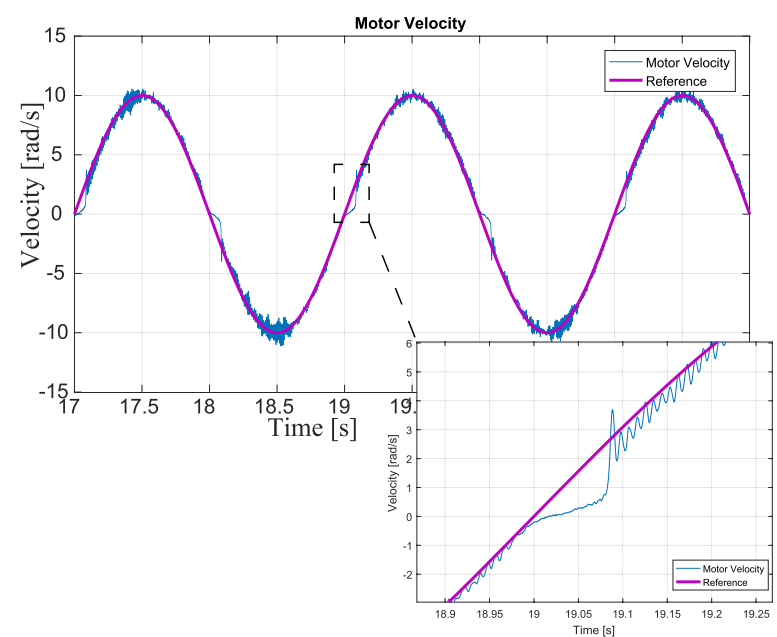

Fig. 5. Velocity response of P-velocity control + ZOMNC (conventional method 1)

The Gopinath method is used to form reduced-order observer for the N-order approximation, which yields the $\mathrm{N}$ order MNC or NOMNC:

$$
\begin{aligned}
& {\left[\begin{array}{c}
\dot{w}_{0} \\
\dot{w}_{1} \\
\vdots \\
\dot{w}_{N-1} \\
\dot{w}_{N}
\end{array}\right]=\left[\begin{array}{ccccc}
\frac{l_{0}}{J_{m n}} & 1 & 0 & \ldots & 0 \\
\frac{l_{1}}{J_{m n}} & 0 & 1 & \ldots & 0 \\
\vdots & & & \ddots & \\
\frac{l_{N-1}}{J_{m n}} & 0 & 0 & \ldots & 1 \\
\frac{l_{N}}{J_{m n}} & 0 & 0 & \ldots & 0
\end{array}\right]\left[\begin{array}{c}
w_{0} \\
w_{1} \\
\vdots \\
w_{N-1} \\
w_{N}
\end{array}\right]} \\
& +\left[\begin{array}{ccc}
\frac{l_{0} l_{0}}{J_{m n}}+l_{1} & -\frac{l_{0} K_{t n}}{J_{m n}} & \frac{l_{0}}{J_{m n} R_{g}} \\
\frac{l_{0} l_{1}}{J_{m n}}+l_{2} & -\frac{l_{1} K_{t n}}{J_{m n}} & \frac{l_{1}}{J_{m n} R_{g}} \\
\vdots & \vdots & \vdots \\
\frac{l_{0} l_{N-1}}{J_{m n}}+l_{N} & -\frac{l_{N-1} K_{t n}}{J_{m n}} & \frac{l_{N-1}}{J_{m n} R_{g}} \\
\frac{l_{0} l_{N}}{J_{m n}} & -\frac{l_{N} K_{t n}}{J_{m n}} & \frac{l_{N}}{J_{m n} R_{q}}
\end{array}\right]\left[\begin{array}{c}
\omega_{m} \\
i_{q}^{r e f} \\
\tau_{s}
\end{array}\right] \\
& {\left[\begin{array}{c}
\hat{\omega}_{m} \\
\hat{\tau}_{d m} \\
\hat{\dot{\tau}}_{d m} \\
\vdots \\
\hat{\tau}_{d m}^{(N)}
\end{array}\right]=\left[\begin{array}{cccc}
0 & 0 & \ldots & 0 \\
1 & 0 & \ldots & 0 \\
0 & 1 & \ldots & 0 \\
\vdots & & \ddots & \\
0 & 0 & \ldots & 1
\end{array}\right]\left[\begin{array}{c}
w_{0} \\
w_{1} \\
w_{2} \\
\vdots \\
w_{N}
\end{array}\right]+\left[\begin{array}{c}
1 \\
l_{0} \\
l_{1} \\
\vdots \\
l_{N}
\end{array}\right] \omega_{m}, \ldots \ldots \ldots}
\end{aligned}
$$

where (6) and (7) are the NOMNC observer and observeroutput equations, respectively. $w_{0} \ldots w_{N}$ are the observer internal states, while $l_{0} \ldots l_{N}$ are the observer gains. The observer gains are designed so that the observer's poles are set to the desired poles $-g_{0} \ldots-g_{N}$. These are calculated by comparing the actual characteristic equation of the NOMNC with the desired one:

$$
s^{N}-\frac{l_{0}}{J_{m n}} s^{N-1}-\ldots-\frac{l_{N-1}}{J_{m n}} s-\frac{l_{N}}{J_{m n}}=\prod_{i=0}^{N}\left(s+g_{i}\right) . \cdots
$$

For the NOMNC, the resulting Q-filter is given as ${ }^{(12)}$ :

$$
Q_{N O M N C}=\frac{k_{0} s^{N}+\ldots+k_{N-1} s+k_{N}}{s^{N+1}+k_{0} s^{N}+\ldots+k_{N-1} s+k_{N}}, \cdots \cdots
$$

where coefficients $k_{0}$ through $k_{N}$ are given by Vieta's formulas ${ }^{(13)}$, as follows: 


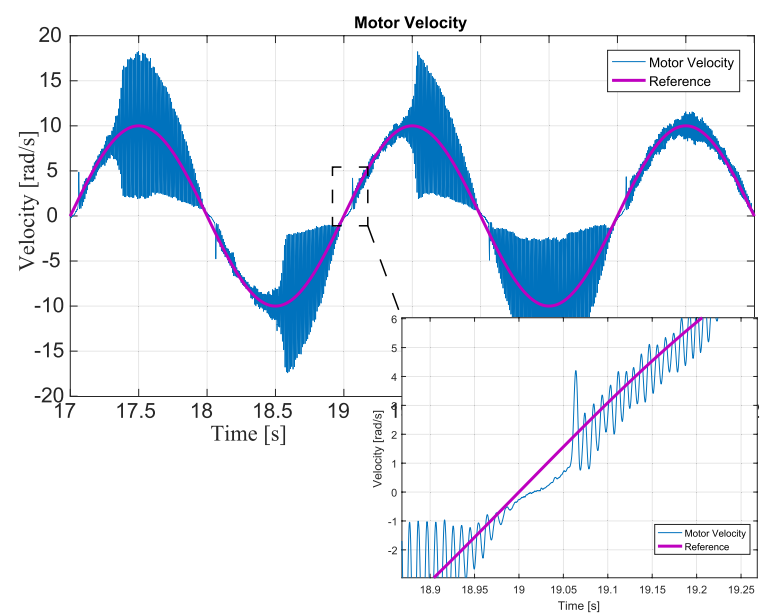

Fig. 6. Velocity response of P-velocity control + First order MNC (conventional method 2)

$$
\left\{\begin{array}{l}
k_{0}=g_{0}+g_{1}+\ldots+g_{N} \\
k_{1}=\left(g_{0} g_{1}+\ldots+g_{0} g_{N}\right)+\left(g_{1} g_{2}+\ldots+g_{1} g_{N}\right)+\ldots+g_{N-1} g_{N} \\
\vdots \\
k_{N}=g_{0} g_{1} \ldots g_{N}
\end{array} .\right.
$$

Examining the Q-filter of the NOMNC confirms that the disturbance rejection is improved. However, increasing the order of the MNC also reduces the robust stability of the system, limiting the maximum bandwidth and making the control system more sensitive to parameter variation.

3.5 Experimental Results: NOMNC Figure 6 shows the experimental results for an $N=1 \mathrm{MNC}$, also known as a first-order MNC (FOMNC). The velocity response has very large vibrations, most probably caused by the reduced robust stability in the system.

Examining the near-zero velocity response reveals that the effects of the stick phenomenon are reduced, but not completely suppressed. However, the velocity overshoot during slipping is larger than in the ZOMNC case. Using an higher order MNC improves suppression of the stick phenomenon, but also worsens stability and the overshoot due to slipping as well.

\section{Proposed Method for Stick Suppression: The VDNSC}

4.1 VDNSC: Velocity-driven N-order Stick Compensator Given that the friction characteristics change between the low and high velocity zones, it makes sense to switch the friction-suppression characteristics between these two regions as well. A combined ZOMNC-FOMNC approach that switches with the velocity is proposed in ${ }^{(14)}$; it successfully reduces the stick phenomenon, but not completely. Switching with an even higher order MNC would endanger the robust stability as the equivalent order of the Qfilter would directly switch between 1 and $N+1$ ( $\mathrm{N}$ being the order of the MNC).

Instead of switching between the order 1 and $N+1 \mathrm{Q}-$ filters, switching could be done successively between order $1,2, \ldots N+1 \mathrm{Q}$-filters to avoid the sudden jump in the order of the resulting filter. A variable relative-degree DOB composed of a series of different-degree Q-filters switched by an error-based switching policy is proposed in ${ }^{(15)}$; This strategy, though, is computation-intensive, as it requires $\mathrm{N}$ different Q-filters whose initial conditions must be adjusted with every switching.

However, recalling that the NOMNC's design is based on a power series in Eq. (5), it is evident that the order of the NOMNC is equal to the number of terms used from the total approximation. By calculating each of the disturbance estimation components and switching them with the motor velocity, the total estimated friction is given as:

$$
\begin{aligned}
\hat{\tau}_{d m}^{V D N S C}= & \hat{\tau}_{d m}^{[0]}+\hat{\tau}_{d m}^{[1]} \alpha_{1}\left(\omega_{m}\right)+\hat{\tau}_{d m}^{[2]} \alpha_{2}\left(\omega_{m}\right)+\ldots \\
& +\hat{\tau}_{d m}^{[N]} \alpha_{N}\left(\omega_{m}\right) . \ldots \ldots \ldots \ldots \ldots \ldots
\end{aligned}
$$

Organizing the terms yields

$$
\hat{\tau}_{d m}^{V D N S C}=\hat{\tau}_{d m}^{[0]}+\sum_{i=1}^{N} \hat{\tau}_{d m}^{[i]} \alpha_{i}\left(\omega_{m}\right) . \cdots \cdots \cdots \cdots \cdots \cdots
$$

This results in the following velocity-driven Q-filter:

$$
Q_{V D N S C}\left(s, \omega_{m}\right)=\frac{g_{0}}{s+g_{0}}+\sum_{i=1}^{N} \frac{g_{i} s^{i}}{\prod_{m=0}^{i}\left(s+g_{m}\right)} \alpha_{i}\left(\omega_{m}\right),
$$

whose order is raised/lowered by turning on/off the switching gains $\alpha_{i}$. The velocity-driven $\mathrm{N}$-order stick compensator (VDNSC) is given by (12). The switching gains $\alpha_{i}$ are designed such that the Q-filter order is high during low speeds, and progressively lowered, one by one, to order 1 as the speed increases; thus, it achieves the same effect as $N+1$ switched Q-filters while using only one. In the following subsection, the procedure for decomposing the NOMNC into its disturbance-estimation components to form a VDNSC will be explained.

4.2 Decomposition of an N-order MNC An alternative form to the Q-filter in Eq. (9) is:

$$
Q_{N O M N C}=\frac{\prod_{i=0}^{N}\left(s+g_{i}\right)-s^{N+1}}{\prod_{i=0}^{N}\left(s+g_{i}\right)} .
$$

This is further rewritten as a complex series by the following fractional decomposition:

$$
\begin{aligned}
& \frac{\prod_{i=0}^{N}\left(s+g_{i}\right)-s^{N+1}}{\prod_{i=0}^{N}\left(s+g_{i}\right)}=\frac{\gamma_{0}}{s+g_{0}}+\frac{\gamma_{1} s}{\left(s+g_{0}\right)\left(s+g_{1}\right)}+\ldots \\
& +\frac{\gamma_{N} s^{N}}{\left(s+g_{0}\right) \ldots\left(s+g_{N}\right)}=\sum_{i=0}^{N} \frac{\gamma_{i} s^{i}}{\prod_{m=0}^{i}\left(s+g_{m}\right)},
\end{aligned}
$$$$
\text { ................ (15) }
$$

where $\gamma_{0} \ldots \gamma_{N}$ are the unknown coefficients of the fractional decomposition. Mathematical induction shows that the coefficients for this partial fraction decomposition are given as

$$
\left\{\begin{array}{l}
\gamma_{0}=g_{0} \\
\gamma_{1}=g_{1} \\
\vdots \\
\gamma_{N}=g_{N}
\end{array}\right.
$$


which are exactly the poles of the NOMNC. Rewriting (15) with this result yields

$$
Q_{N O M N C}=\sum_{i=0}^{N} \frac{g_{i} s^{i}}{\prod_{m=0}^{i}\left(s+g_{m}\right)}
$$

Consequently, the total estimated friction is expressed as a combination of $N+1$ terms:

$$
\hat{\tau}_{d m}^{N O M N C}=\tau_{d m} \sum_{i=0}^{N} \frac{g_{i} s^{i}}{\prod_{m=0}^{i}\left(s+g_{m}\right)}=\sum_{i=0}^{N} \hat{\tau}_{d m}^{[i]}, \cdots
$$

where $\hat{\tau}_{d m}^{[i]}$ is defined as the $\mathrm{i}$-th order estimation component, which is given as

$$
\hat{\tau}_{d m}^{[i]}=\frac{g_{i} s^{i}}{\prod_{m=0}^{i}\left(s+g_{m}\right)} \tau_{d m} .
$$

From (18), it is clear that the Q-filter order may be changed by choosing how many disturbance components are taken. Taking $N=0$ and $N=1$, for example, yields:

$$
\begin{aligned}
& (\mathrm{N}=0) \quad \hat{\tau}_{d m}^{[0]}=\tau_{d m} \frac{g_{0}}{s+g_{0}} \ldots \ldots \ldots \ldots \ldots \ldots \\
& (\mathrm{N}=1) \quad \hat{\tau}_{d m}^{[0]}+\hat{\tau}_{d m}^{[1]}=\tau_{d m} \frac{\left(g_{0}+g_{1}\right) s+g_{0} g_{1}}{\left(s+g_{0}\right)\left(s+g_{1}\right)},
\end{aligned}
$$

where (20) and (21) correspond to a ZOMNC and an FOMNC, respectively.

The $\hat{\tau}_{d m}^{[i]}$ estimation components, however, are not directly calculable from (7), as only the total estimated friction and its derivatives $\hat{\tau}_{d m}, \hat{\bar{\tau}}_{d m} \ldots \hat{\tau}_{d m}^{(N)}$ are available. Taking (6) and (7) and assuming no parameter variation, the estimated friction torque and its derivatives are given as:

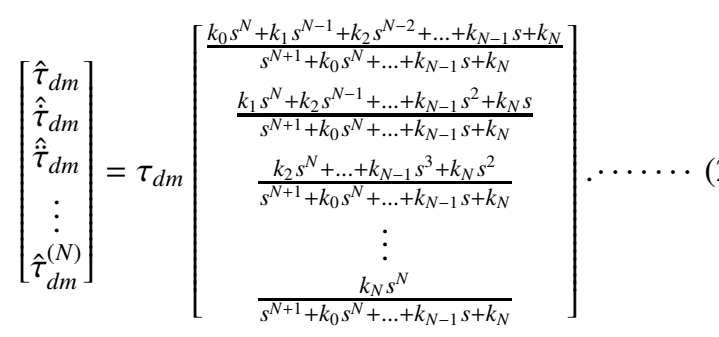

Observing that the denominator of the transfer function for $\hat{\tau}_{d m}, \hat{\hat{\tau}}_{d m} \ldots \hat{\tau}_{d m}^{(N)}$ is the same, the above equation is rewritten as

$$
\begin{aligned}
& {\left[\begin{array}{c}
\hat{\tau}_{d m} \\
\hat{\tilde{\tau}}_{d m} \\
\hat{\tilde{\tau}}_{d m} \\
\vdots \\
\hat{\tau}_{d m}^{(N)}
\end{array}\right]=\frac{\tau_{d m}}{\prod_{i=0}^{N}\left(s+g_{i}\right)} \boldsymbol{\varphi}\left[\begin{array}{c}
s^{N} \\
s^{N-1} \\
s^{N-2} \\
\vdots \\
s^{0}
\end{array}\right] \ldots \ldots \ldots} \\
& \boldsymbol{\varphi}=\left[\begin{array}{cccccc}
k_{0} & k_{1} & k_{2} & \ldots & k_{N-1} & k_{N} \\
k_{1} & k_{2} & k_{3} & \ldots & k_{N} & 0 \\
\vdots & \vdots & \vdots & \vdots & \vdots & \vdots \\
k_{N-1} & k_{N} & 0 & \ldots & 0 & 0 \\
k_{N} & 0 & 0 & \ldots & 0 & 0
\end{array}\right],
\end{aligned}
$$

where $\varphi$ is the coefficient matrix for the numerators of each of the transfer functions from the real value $\tau_{d m}$ to the estimated friction and its derivatives $\hat{\tau}_{d m}, \hat{\bar{\tau}}_{d m} \ldots \hat{\tau}_{d m}^{(N)}$. Meanwhile, the estimation components $\hat{\tau}_{d m}^{[0]} \ldots \hat{\tau}_{d m}^{[N]}$ is rewritten as

$$
\left[\begin{array}{c}
\hat{\tau}_{d m}^{[0]} \\
\hat{\tau}_{d m}^{[1]} \\
\hat{\tau}_{d m}^{[2]} \\
\vdots \\
\hat{\tau}_{d m}^{[N]}
\end{array}\right]=\tau_{d m}\left[\begin{array}{c}
\frac{g_{0}}{s+g_{0}} \\
\frac{g_{1} s}{\left(s+g_{0}\right)\left(s+g_{1}\right)} \\
\frac{g_{2} s^{2}}{\left(s+g_{0}\right)\left(s+g_{1}\right)\left(s+g_{2}\right)} \\
\vdots \\
\frac{g_{N} s^{N}}{\left(s+g_{0}\right)\left(s+g_{1}\right) \ldots\left(s+g_{N}\right)}
\end{array}\right]
$$

Algebraic manipulation is then employed to (25) so that each estimation component has $\prod_{i=0}^{N}\left(s+g_{i}\right)$ as denominator. Doing this for the first two terms, for example, results as

$$
\begin{aligned}
& \frac{g_{0}}{s+g_{0}}=\frac{g_{0}\left(s+g_{1}\right)\left(s+g_{2}\right) \ldots\left(s+g_{N}\right)}{\left(s+g_{0}\right)\left(s+g_{1}\right) \ldots\left(s+g_{N}\right)} \ldots \ldots . \\
& \frac{g_{1} s}{\left(s+g_{0}\right)\left(s+g_{1}\right)}=\frac{g_{1} s\left(s+g_{2}\right)\left(s+g_{3}\right) \ldots\left(s+g_{N}\right)}{\left(s+g_{0}\right)\left(s+g_{1}\right) \ldots\left(s+g_{N}\right)} .
\end{aligned}
$$

Applying this procedure to all terms of (25), they are rewritten in a manner similar to (24), as follows:

$$
\begin{gathered}
{\left[\begin{array}{c}
\hat{\tau}_{d m}^{[0]} \\
\hat{\tau}_{d m}^{[1]} \\
\hat{\tau}_{d m}^{[2]} \\
\vdots \\
\hat{\tau}_{d m}^{[N]}
\end{array}\right]=\frac{\tau_{d m}}{\prod_{i=0}^{N}\left(s+g_{i}\right)} \varphi_{e c}\left[\begin{array}{c}
s^{N} \\
s^{N-1} \\
s^{N-2} \\
\vdots \\
s^{0}
\end{array}\right] \ldots \ldots \ldots \ldots \ldots(28)} \\
\boldsymbol{\varphi}_{e c}=\left[\begin{array}{cccccc}
g_{0} & g_{0} k_{0}^{j=1} & g_{0} k_{1}^{j=1} & \ldots & g_{0} k_{N-2}^{j=1} & g_{0} k_{N-1}^{j=1} \\
g_{1} & g_{1} k_{0}^{j=2} & g_{1} k_{1}^{j=2} & \ldots & g_{1} k_{N-2}^{j=2} & 0 \\
\vdots & \vdots & \vdots & \vdots & \vdots & \vdots \\
g_{N-1} & g_{N-1} k_{0}^{j=N} & \ldots & 0 & 0 & 0 \\
g_{N} & 0 & \ldots & 0 & 0 & 0
\end{array}\right],
\end{gathered}
$$

where $\boldsymbol{\varphi}_{e c}$ is the coefficient matrix for the numerators of the transfer functions from the real value of friction $\tau_{d m}$ to each of the estimation components $\hat{\tau}_{d m}^{[0]}, \hat{\tau}_{d m}^{[1]}, \ldots \hat{\tau}_{d m}^{[N]} . k_{q}^{j=m}$ stands for the q-th coefficient of the $\prod_{j=m}^{N}\left(s+g_{j}\right)$ polynomial, given as:

$$
\prod_{j=m}^{N}\left(s+g_{j}\right)=s^{N-m+1}+k_{0}^{j=m} s^{N-m}+k_{1}^{j=m} s^{N-m-1}+\ldots+k_{N-m}^{j=m}
$$

Both $\varphi$ and $\varphi_{e c}$ form triangular matrices with non-zero main diagonal entries, and are thus invertible. A transformation matrix $\boldsymbol{T}$ is then defined as:

$$
\boldsymbol{T}=\boldsymbol{\varphi}_{e c} \boldsymbol{\varphi}^{-1}
$$

Finally, the estimation components are estimated as:

$$
\left[\begin{array}{c}
\hat{\tau}_{d m}^{[0]} \\
\hat{\tau}_{d m}^{[1]} \\
\hat{\tau}_{d m}^{[2]} \\
\vdots \\
\hat{\tau}_{d m}^{[N]}
\end{array}\right]=\boldsymbol{T}\left[\begin{array}{c}
\hat{\tau}_{d m} \\
\dot{\hat{\tau}}_{d m} \\
\ddot{\hat{\tau}}_{d m} \\
\vdots \\
\hat{\tau}_{d m}^{(N)}
\end{array}\right]
$$




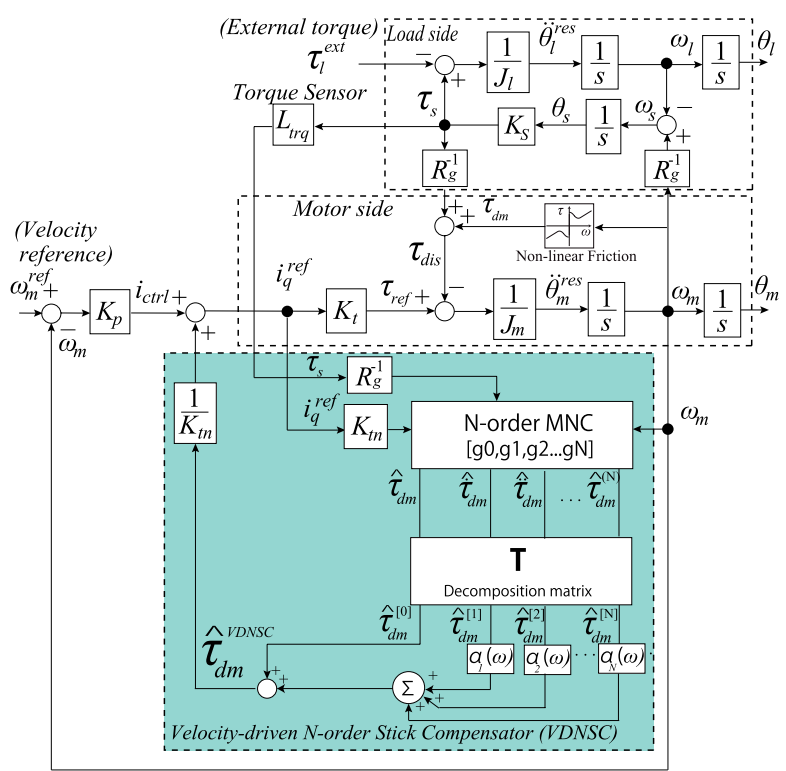

Fig. 7. Block diagram of proposed VDNSC (proposed method 1)

Thus, the decomposition of the NOMNC into its estimation components is done by applying $\boldsymbol{T}$ to the $\mathrm{N}$-order MNC observer-output equation (Eq. (7)). Figure 7 shows the block diagram of the VDNSC combined with the P-velocity control system.

4.3 Design Example Based on the methodology explained in the previous section, a VDNSC with maximum order $N=3$ and four arbitrary, stable poles, $-g_{0} \ldots-g_{3}$ was designed. For $N=3$, the transformation matrix $\boldsymbol{T}$ is given as

$$
\boldsymbol{T}=\left[\begin{array}{cccc}
1 & -\frac{1}{g_{0}} & \frac{1}{g_{0}^{2}} & -\frac{1}{g_{0}^{3}} \\
0 & \frac{1}{g_{0}} & -\frac{g_{0}+g_{1}}{g_{0}^{2} g_{1}} & \frac{g_{0}^{2}+g_{0} g_{1}+g_{1}^{2}}{g_{0}^{3} g_{1}^{2}} \\
0 & 0 & \frac{1}{g_{0} g_{1}} & -\frac{g_{0} g_{1}+g_{0} g_{2}+g_{1} g_{2}}{g_{0}^{2} g_{1} g_{2}} \\
0 & 0 & 0 & \frac{1}{g_{0} g_{1} g_{2}}
\end{array}\right]
$$

Applying the transformation to Eq. (7) results in the following output equation:

$\left[\begin{array}{c}\hat{\omega}_{m} \\ \hat{\tau}_{d m}^{[0]} \\ \hat{\tau}_{d m}^{[1]} \\ \hat{\tau}_{d m}^{[2]} \\ \hat{\tau}_{d m}^{[3]}\end{array}\right]=\left[\begin{array}{cccc}0 & 0 & 0 & 0 \\ 1 & -\frac{1}{g_{0}} & \frac{1}{g_{0}^{2}} & -\frac{1}{g_{0}^{3}} \\ 0 & \frac{1}{g_{0}} & -\frac{g_{0}+g_{1}}{g_{0}^{2} g_{1}} & \frac{g_{0}^{2}+g_{0} g_{1}+g_{1}^{2}}{g_{0}^{3} g_{1}^{2}} \\ 0 & 0 & \frac{1}{g_{0} g_{1}} & -\frac{g_{0} g_{1}+g_{0} g_{2}+g_{1} g_{2}}{g_{0}^{2} g_{1}^{2} g_{2}} \\ 0 & 0 & 0 & \frac{1}{g_{0} g_{1} g_{2}}\end{array}\right]\left[\begin{array}{l}w_{0} \\ w_{1} \\ w_{2} \\ w_{3} \\ w_{4}\end{array}\right]+\left[\begin{array}{c}1 \\ -J_{m n} g_{0} \\ -J_{m n} g_{1} \\ -J_{m n} g_{2} \\ -J_{m n} g_{3}\end{array}\right] \omega_{m}$.

Applying velocity-dependent switching gains $\alpha_{1} \ldots \alpha_{3}$ results in the following total estimated friction:

$$
\hat{\tau}_{d m}^{V D N S C}=\hat{\tau}_{d m}^{[0]}+\hat{\tau}_{d m}^{[1]} \alpha_{1}\left(\omega_{m}\right)+\hat{\tau}_{d m}^{[2]} \alpha_{2}\left(\omega_{m}\right)+\hat{\tau}_{d m}^{[3]} \alpha_{3}\left(\omega_{m}\right) .
$$$$
\text { ................ (35) }
$$

Feeding this estimated friction value back to the motor results in the following velocity-dependent disturbancerejection characteristic:

$$
1-Q_{V D N S C}\left(s, \omega_{m}\right)=\frac{s\left(s^{3}+\phi_{2}\left(\omega_{m}\right) s^{2}+\phi_{1}\left(\omega_{m}\right) s+\phi_{0}\left(\omega_{m}\right)\right)}{\left(s+g_{0}\right)\left(s+g_{1}\right)\left(s+g_{2}\right)\left(s+g_{3}\right)},
$$

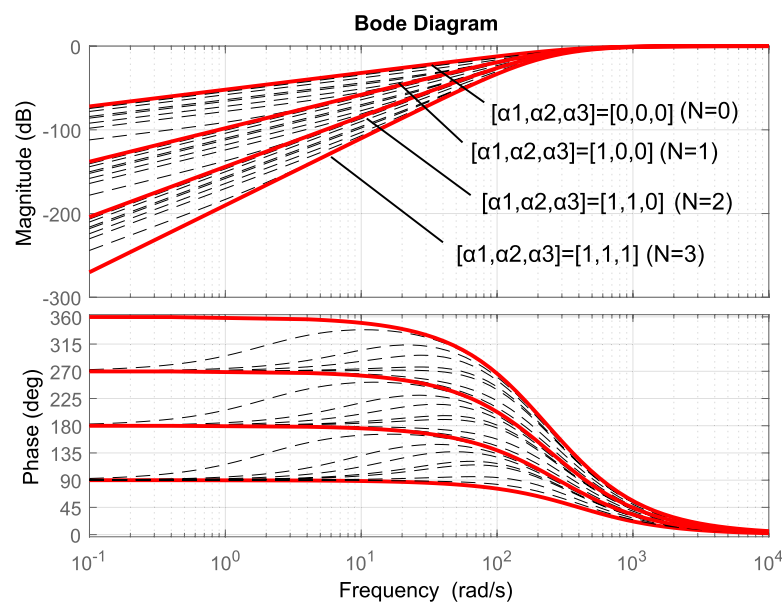

Fig. 8. Disturbance rejection characteristic of designed $N=3$ VDNSC as the switching gains $\alpha_{1} \ldots \alpha_{3}$ are varied (poles set to $[400,200,200,200] \mathrm{rad} / \mathrm{s}$ )

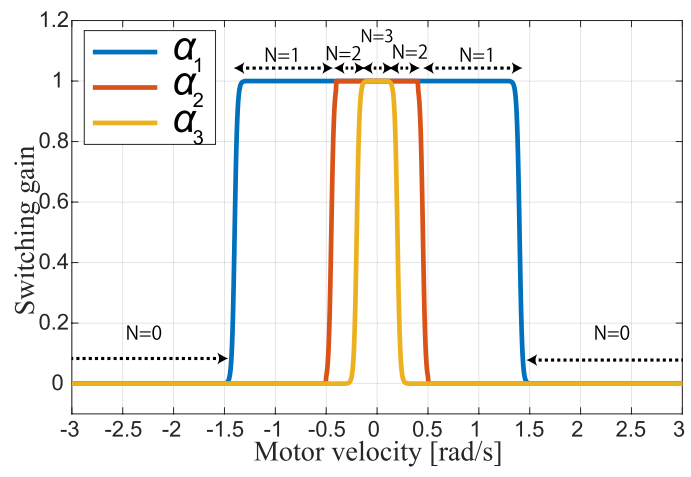

Fig. 9. Switching pattern for VDNSC

where $\phi_{2}$ to $\phi_{0}$ are velocity-dependent coefficients, given as

$$
\begin{aligned}
& \phi_{2}=g_{1}\left(1-\alpha_{1}\left(\omega_{m}\right)\right)+g_{2}\left(1-\alpha_{2}\left(\omega_{m}\right)\right) \\
& +g_{3}\left(1-\alpha_{3}\left(\omega_{m}\right)\right) \\
& \phi_{1}=\left(g_{1} g_{2}+g_{1} g_{3}\right)\left(1-\alpha_{1}\left(\omega_{m}\right)\right)+g_{2} g_{3}\left(1-\alpha_{2}\left(\omega_{m}\right)\right) \\
& \phi_{0}=g_{1} g_{2} g_{3}\left(1-\alpha_{1}\left(\omega_{m}\right)\right) .
\end{aligned}
$$

Figure 8 shows how the disturbance-rejection characteristic varies as the switching gains are varied. The disturbance rejection of the VDNSC is increased by turning the switching gains to 1 , and decreased by gradually turning them to 0 .

The switching patterns are designed such that the VDNSC order is at its maximum $(N=3)$ around zero-speed, and at its minimum $(N=0)$ as the motor accelerates and enters the kinetic friction region. The proposed design implemented in this paper is shown in Fig. 9.

4.4 Experimental Results: VDNSC Figure 10 shows the experimental results using the proposed VDNSC. The braking effect due to the stick phenomenon is greatly suppressed, while keeping a stable response at higher speeds. However, there is still the effect of the slip phenomenon, as seen in the velocity overshoot that occurs after the motor turning direction has changed.

5. Proposed Method for Slip Suppression: The Slip Suppressor

5.1 Slip Phenomenon The slip phenomenon occurs 


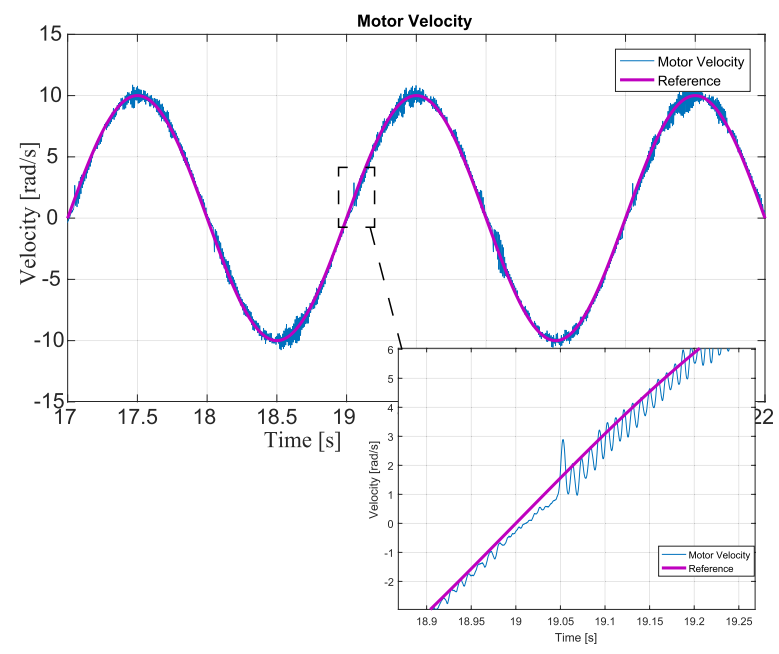

Fig. 10. Velocity response of P-velocity control + VDNSC (proposed method 1)

after the motor overcomes pre-sliding friction and the contact between the mechanical components is dominated by the mixed lubrication regime, in which the oil film begins to form between the contacting surfaces and the friction coefficient dramatically decreases ${ }^{(16)}$.

Based on the steady-state friction curve model shown in Fig. 2, the friction value over the slip zone is approximated as follows:

$$
\tau_{\text {slip }}=\tau_{\text {static }}-D_{\text {slip }}\left(\omega_{m}\right), \cdots
$$

where $\tau_{\text {static }}$ is the static friction, and the subtracting term $D_{\text {slip }}$ describes the velocity-proportional decrease in friction as the motor slips. Assuming that as the motor overcomes static friction and slips the VDNSC order is $N=0$, i.e., a simple ZOMNC, the dynamics for this zone are described as follows:

$$
J_{m} \dot{\omega}_{m}=K_{t} i_{c t r l}-\frac{\tau_{s}}{R_{g}}-\tau_{\text {slip }}\left(1-Q_{Z O M N C}(s)\right) . \cdots
$$

Expanding the previous equation yields:

$$
J_{m} \dot{\omega}_{m}=K_{t} i_{c t r l}-\frac{\tau_{s}}{R_{g}}-\left(\tau_{\text {static }}-D_{\text {slip }}\left(\omega_{m}\right)\right) \frac{s}{s+g_{0}}, \cdots
$$

As the motor starts to move, $\tau_{\text {static }}$ is already compensated by the VDNSC, leaving only the negative damping term. This results in

$$
J_{m} \dot{\omega}_{m}=K_{t} i_{c t r l}-\frac{\tau_{s}}{R_{g}}+D_{s l i p}\left(\omega_{m}\right) \frac{s}{s+g_{0}} . \cdots \cdots \cdots
$$

As seen in Fig. 1, the transition from static to kinetic friction occurs very quickly. This means that the friction during the slip zone includes high-frequency components that cannot be totally suppressed by the VDNSC. The negative damping also destabilizes the system, rendering the VDNSC useless.

5.2 High-frequency Damping for Slip Suppression One possible method for suppressing the remaining highfrequency negative damping components over the slip zone is to apply positive high-frequency damping. The highfrequency damping feedback term is given as

$$
\tau_{H F D}=K_{v} \frac{s}{s+g_{d m p}} \omega_{m}
$$

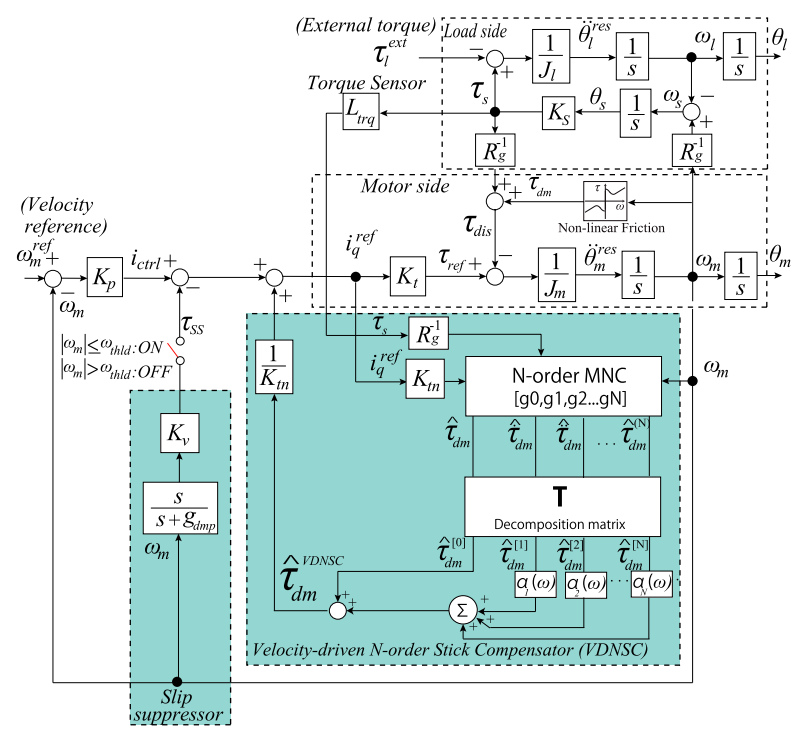

Fig. 11. Block diagram of the proposed VDNSC + slip suppressor system (proposed method 2)

where $K_{v}$ is the damping gain and $g_{d m p}$ is the cutoff frequency of the high-pass filter. Applying high-frequency damping changes the motor dynamics as follows:

$J_{m} \dot{\omega}_{m}=K_{t} i_{c t r l}-\frac{\tau_{s}}{R_{g}}+D_{\text {slip }}\left(\omega_{m}\right) \frac{s}{s+g_{0}}-K_{t} K_{v} \frac{s}{s+g_{d m p}} \omega_{m}$.

The $K_{v}$ and $g_{d m p}$ parameters were tuned experimentally to suppress the slip phenomenon.

5.3 Slip Suppressor The high-frequency damping is also interpreted as an artificial change in the system's inertia, and applying it at all times may affect the performance of the applied control system. Meanwhile, effectively detecting when the motor slips is difficult. The proposed solution in this paper is to switch the high-frequency damping, applying it only through a very short range of velocities, as given by the following equation:

$$
\tau_{S S}=\left\{\begin{array}{ll}
K_{v} \frac{s}{s+g_{d m p}} \omega_{m}, & \text { if }\left|\omega_{m}\right| \leq \omega_{\text {thld }} \\
0, & \text { if }\left|\omega_{m}\right|>\omega_{\text {thld }}
\end{array}, .\right.
$$

where $\tau_{S S}$ is the output of the slip suppressor.

The velocity threshold $\omega_{\text {thld }}$ parameter was selected experimentally to cover both the pre-sliding and slip zones. By designing it in this manner, the high-frequency damping prevents not only the slip phenomenon itself, but also sudden movement from the quick switching of the VDNSC. The total velocity range of operation is made as small as possible so it does not interfere with the controller applied to the motor.

The complete stick-slip rejection system including the VDNSC and the slip suppressor is shown in Fig. 11.

5.4 Experimental Results: VDNSC + Slip Suppressor Figure 12 shows the results for the P-velocity control and the combined VDNSC and slip suppressor system. The velocity overshoot during the velocity-crossing was reduced by $50 \%$ compared to the VDNSC-only case, while also suppressing the stick phenomenon.

\section{Discussion of Results}

Figure 13 shows a comparison of the current and velocity 


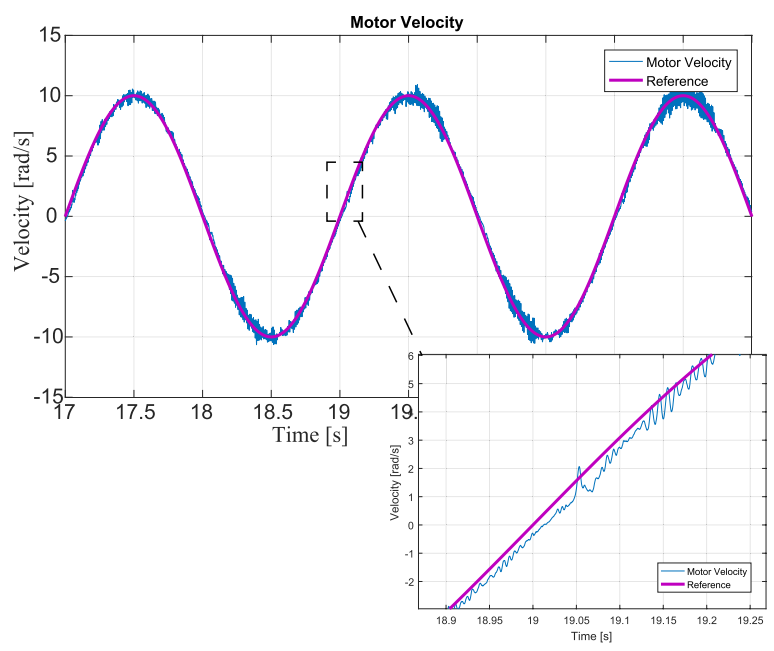

Fig. 12. Velocity response of P-velocity control + VDNSC + Slip suppressor (proposed method 2)
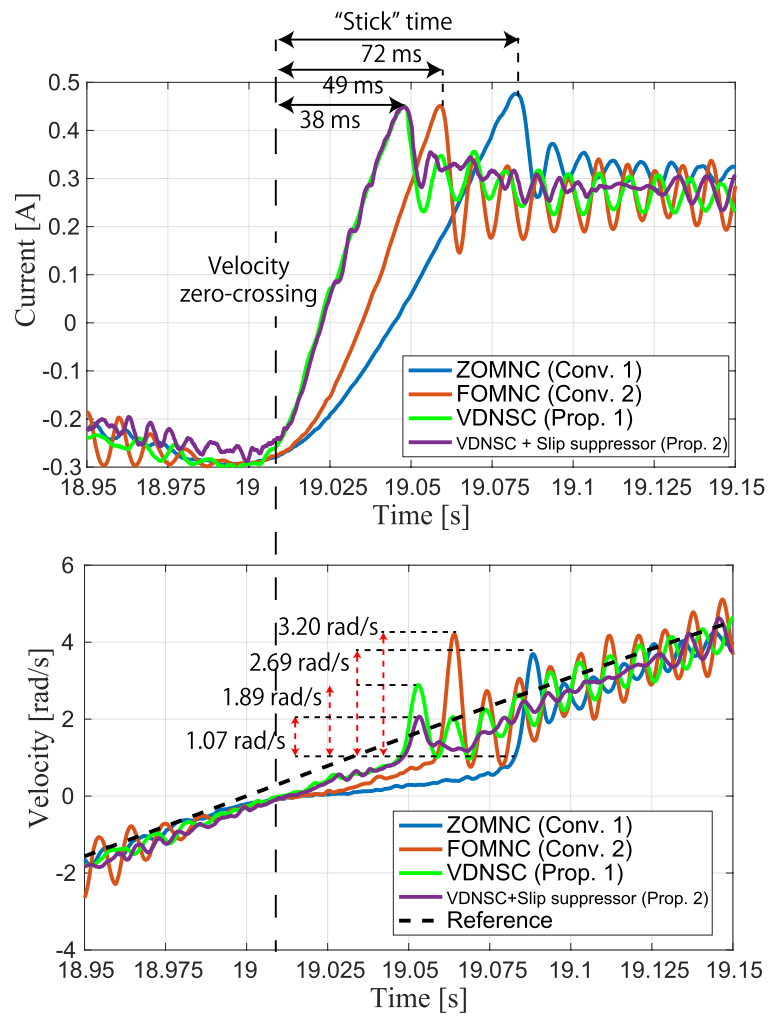

Fig. 13. Comparison of stick time and slip overshoot over velocity zero-crossings

responses for the four friction compensation methods tested in this study around the velocity zero-crossing that occurs at $t=19$ seconds. The "stick" time (in this case, the time taken from the actual zero-crossing until the slipping of the motor) is the smallest for the proposed method. Moreover, the current and velocity slopes of the proposed method are steeper during the sticking, indicating that the motor is more effectively counteracting the dry friction.

In addition, the velocity response shows that the proposed method has the smallest velocity overshoot when slipping, resulting in a $66.6 \%$ reduction compared to the FOMNC, which has the largest overshoot.

6.1 Harmonic Analysis of Velocity Response In

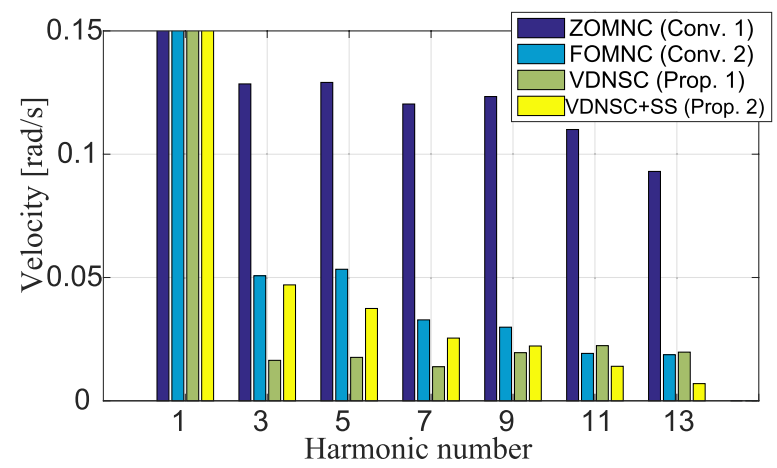

Fig. 14. Calculated harmonics for the velocity response of each of the tested methods $\left(f_{1}=0.5 \mathrm{~Hz}\right)$

Table 2. Numerical comparison of the tested friction suppression methods

\begin{tabular}{|c|c|c|}
\hline Friction suppression method & Stick-time [ms] & Slip overshoot [rad/s] \\
\hline ZOMNC (Conventional 1) & 72 & 2.69 \\
\hline FOMNC (Conventional 2) & 49 & 3.20 \\
\hline VDNSC (Proposed 1) & 38 & 1.89 \\
\hline VDNSC + Slip Suppressor (Proposed 2) & 38 & 1.07 \\
\hline
\end{tabular}

Table 3. THD comparison of tested methods

\begin{tabular}{|c|c|}
\hline Friction suppression method & THD (\%) \\
\hline ZOMNC (Conventional 1) & 2.90 \\
\hline FOMNC (Conventional 2) & 0.90 \\
\hline VDNSC (Proposed 1) & 0.45 \\
\hline VDNSC + Slip Suppressor (Proposed 2) & 0.71 \\
\hline
\end{tabular}

order to analyze and quantify the degree of distortion due to nonlinear friction in the velocity response, frequency analysis was performed. Figure 14 shows the first seven odd harmonics, spanning from 0.5 to $7.5 \mathrm{~Hz}$. Using the fact that the velocity reference is a sinusoidal signal, THD calculations are employed to quantify distortion due to nonlinearity in the same manner as an electrical system, with the formula in this case given as

$$
\operatorname{THD}(\%)=\frac{\sqrt{\sum_{i=2}^{N} \omega_{m_{i}}^{2}}}{\omega_{m_{1}}} \times 100 \%,
$$

where $\omega_{m_{i}}$ stands for the i-th velocity harmonic, and $\omega_{m_{1}}$ is the fundamental frequency velocity component. Applying this calculation for the first seven odd harmonics yields the results in Table 3. The VDNSC alone yields the lowest THD, followed by the VDNSC and slip suppressor combination. Adding the slip suppressor increases harmonic distortion, but it still keeps it below the numbers of the conventional methods, offering a THD four times smaller than the ZOMNC.

\section{Conclusion}

This paper proposed a stick-slip suppression method for oil-seal-mounted geared motors based on a velocity-driven $\mathrm{N}$-order stick compensator and a slip suppressor. As shown in Table 2, the proposed VDNSC and slip suppressor proved to be more effective than other conventional methods, e.g., the Zero-order MNC and the $\mathrm{N}$-order $\mathrm{MNC}$, with a sticktime reduction of $47 \%$ when compared to the ZOMNC, and a slip-overshoot reduction of around $66.6 \%$, compared to the FOMNC. THD calculations also confirmed that the proposed method gives a much more linear response, offering a $0.45 \%$ 
when using only the VDNSC and $0.71 \%$ using the combined VDNSC and slip suppressor, reducing THD to four times less than that of the conventional ZOMNC.

By switching the disturbance rejection between the nonlinear friction and kinetic friction regimes, the proposed method is able to compensate for strong nonlinear friction on low velocities while maintaining stability for higher velocities.

The proposed method also has the benefit of not requiring any explicit friction model in its implementation; it only needs to determine the velocity range in which the nonlinear friction arises.

\section{References}

(1) H. Nakamura, K. Ohishi, Y. Yokokura, N. Kamiya, T. Miyazaki, and A. Tsukamoto: "Force Sensorless Fine Force Control Based on Notch-Type Friction-Free Disturbance Observers", in IEEJ Journal of Industry Applications, Vol.7, No.2, pp.117-126 (2018)

( 2 ) L. Freidovich, A. Robertsson, A. Shiriaev, and R. Johansson: "LuGre-ModelBased Friction Compensation", in IEEE Transactions on Control Systems Technology, Vol.18, No.21, pp.194-200 (2010)

( 3 ) C. Canudas de Wit, H. Olsson, K.J. Astrom, and P. Lischinsky: "A New Model for Control of Systems with Friction", in IEEE Transactions on Automatic Control, Vol.40, No.3, pp.419-425 (1995)

( 4 ) D.H. Lee and J.W. Ahn: "Dual speed control scheme of servo drive system for a nonlinear friction compensation", in IEEE Transactions on Power Electronics, Vol.23, No.2, pp.959-965 (2008)

( 5 ) K.A.J. Verbert, R. Tóth, and R. Babuška: "Adaptive Friction Compensation: A Globally Stable Approach", inIEEE/ASME Transactions on Mechatronics, Vol.21, No.1, pp.351-363 (2016)

( 6 ) M. Iwasaki, T. Shibata, and N. Matsui: "Disturbance-Observer-Based Nonlinear Friction Compensation in Table Drive System", in IEEE/ASME Transactions on Mechatronics, Vol.4, No.1, pp.3-8 (1999)

( 7 ) G. Zhang and J. Furusho: "Control of Robot Arms Using Joint Torque Sensors", in IEEE Transactions on Control Systems Magazine, Vol.18, No.1, pp.48-55 (1998)

( 8 ) Y. Kawai, Y. Yokokura, K. Ohishi, and T. Miyazaki: "Smooth Human Interaction Control using Torsion Torque Controller and Motor-side Normalization Compensator Focusing on Back-forward Drivability", in IEEJ Journal of Industry Applications, Vol.8, No.2, pp.322-333 (2019)

( 9 ) K. Ohnishi, M. Shibata, and T. Murakami: "Motion Control for Advanced Mechatronics", in IEEE/ASME Transactions on Mechatronics, Vol.1, No.1, pp.56-67 (1996)

(10) J. Padron, Y. Yokokura, and K. Ohishi: "Stick-slip suppression in geared motor by speed-dependent switching of high-order Motor-side Normalization Compensator components", in The Papers of Technical Meeting on "Mechatronics Control", IEEJ, pp.41-46 (2018)

(11) T.T. Phuong, K. Ohishi, Y. Yokokura, and C. Mitsantisuk: "FPGA-based high-performance force control system with friction-free and noise-free force observation", in IEEE Trans. on Industrial Electronics, Vol.61, No.2, pp.9941008 (2014)

(12) K. Yamada, S. Komada, M. Ishida, and T. Hori: "Analysis and classical control design of servo system using high order disturbance observer", in IEEE IECON'97, pp.4-9 (1997)

(13) Encyclopedia of Mathematics, "Viète theorem" [Online]. Available: http:// www.encyclopediaofmath.org/index.php?title=Vi\%C3\%A8te_theorem\&oldi $\mathrm{d}=23172$ [Accessed Apr. 19, 2019]

(14) J. Padron, Y. Yokokura, and K. Ohishi: "Suppression of stick-slip phenomenon on geared motor with oil seal using a sensitivity-variable MNC", in The 36th Annual Conference of the Robotics Society of Japan, pp.1-4 (2018)

(15) Y.Q. Chen, B.M. Vinagre, and I. Podlubni: "Fractional order disturbance observer for vibration suppression", in Nonlinear Dynamics, Vol.38, No.1-4 pp.355-367 (2004)
(16) D.J. Schipper: "Transitions in the lubrication of concentrated contacts", Ph.D. thesis, University of Twente, The Netherlands (1988)

Juan Padron (Student Member) received the B.E. degrees in elec-

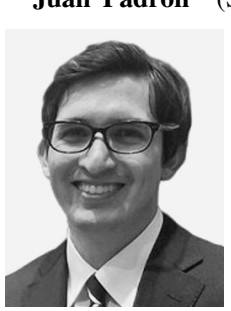
tronics engineering from Universidad Simon Bolivar, Caracas, Venezuela, in 2016. Now he is a candidate of the M.E. degree in Electrical, Electronics and Information Engineering. His research interests include motion control, robot-human interaction and nonlinear friction compensation. He is a member of the Institute of Electrical Engineers of Japan (IEEJ) and the IEEE Industrial Electronics Society (IEEE IES).

Kiyoshi Ohishi (Fellow) received the B.E., M.E., and Ph.D. degrees

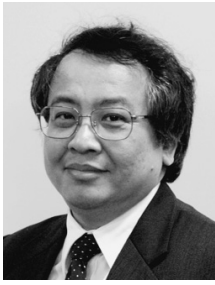
in electrical engineering from Keio University, Yokohama, Japan, in 1981, 1983, and 1986, respectively. From 1986 to 1993, Prof. Ohishi was an Associate Professor with Osaka Institute of Technology, Osaka, Japan. Since 1993, he has been with Nagaoka University of Technology, Niigata, Japan. He became a Professor in 2003. His research interests include motion control, mechatronics, robotics and power electronics. He received twice "IEEJ Distinguished $\mathrm{Pa}$ per Award" from IEEJ in 2002 and 2009, respectively. He is also an IEEE Fellow member. From 2004, he has been an AdCom Member of IEEE Industrial Electronic Society (IES). He received the Oustanding Paper Awards at IECON'85 from IEEE IES. Moreover, he received the Best Paper Awards at IECON2002 and IECON2004 from IEEE IES. He was a General chair of IEEE IECON2015 and IEEE AMC2010, AMC2016 and AMC2018.

Yuki Yokokura

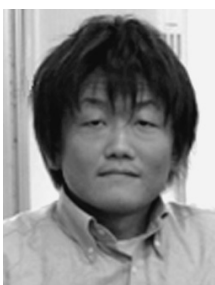

(Member) received the B.E. and M.E. degrees in electrical engineering from Nagaoka University of Technology, Niigata, Japan, in 2007 and 2009, respectively. In 2011, he received the Ph.D. degree in integrated design engineering from Keio University, Yokohama, Japan. From 2010 to 2011, he was a JSPS (Japan Society for the Promotion of Science) Research Fellow (DC2 and PD). He was a Visiting Fellow at Keio University, and a Postdoctoral Fellow at Nagaoka University of Technology in 2011. Since 2012, he has been an Assistant Professor with Nagaoka University of Technology. His research interests include motion control, motor drive, power electronics, and real-world haptics.

Toshimasa Miyazaki (Member) received the B.E. and M.E., and

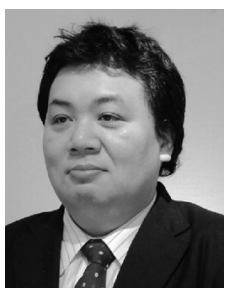
$\mathrm{Ph} . \mathrm{D}$. degrees all in electrical engineering, from Nagaoka University of Technology, Niigata, Japan, in 1994, 1996, and 1999, respectively. From 1999 to 2010, he was an Associate Professor with Nagaoka National College of Technology, Niigata, Japan. Since 2010, he has been an Associate Professor with Nagaoka University of Technology, Niigata, Japan. His research interests include motion control and powerelectronics. He is a member of the IEEE Industrial Electronics Society (IEEE IES) and the Institute of Electrical Engineers of Japan (IEEJ). 